\title{
Effects of dexamethasone on peritumoural oedematous brain: a DT-MRI study
}

\author{
S Sinha, M E Bastin, J M Wardlaw, P A Armitage, I R Whittle
}

J Neurol Neurosurg Psychiatry 2004;75:1632-1635. doi: 10.1136/jnnp.2003.028647

Objectives: Glucocorticoids (dexamethasone) are thought to reduce peritumoural brain oedema by decreasing the permeability of neoplastic capillaries and/or enhancing the clearance of extracellular water. Diffusion tensor magnetic resonance imaging (DT-MRI) was used to measure the water diffusion parameters of oedematous and normal brain in a group of patients with intracranial tumours before and after steroid treatment.

Methods: Fifteen patients with intracranial tumours (seven with high-grade glioma, four with metastatic carcinoma and four with meningioma) were examined before and $48-72 \mathrm{~h}$ after dexamethasone treatment $(16 \mathrm{mg} /$ day). The mean diffusivity $(\langle D>)$ and fractional anisotropy (FA) were measured for oedematous brain and apparently normal contralateral white matter before and after steroid therapy. Results: In all three patient groups there was a significant decrease in $<D>$ of oedematous brain after steroid treatment $(p<0.01)$. There was no significant change in FA of oedematous brain after treatment in any of the three groups. There was also no significant change in either $\langle D\rangle$ or FA of apparently normal contralateral white matter after treatment.

Conclusion: These data indicate that dexamethasone produces a localised reduction in the magnitude of extracellular water molecule mobility, and hence water content, in peritumoural oedematous brain. Furthermore, the magnitude of these changes is similar for both intra- and extra-axial tumours.

1 patients with malignant cerebral tumours, the signs and symptoms are due to not only the location and mass effect of the tumour, but also the associated peritumoural oedema. Following several studies that documented a decrease in both surgical morbidity and mortality following glucocorticoid (dexamethasone) treatment, ${ }^{12}$ steroid therapy is now routinely used in the management of patients with brain tumours and peritumoural oedema. Although their modes of action are poorly understood, steroids are thought to play a major role in decreasing oedema formation by reducing the permeability of neoplastic capillaries and/or enhancing the clearance of extracellular water. ${ }^{3}$

The effects of glucocorticoids on water abnormalities in peritumoural brain can be assessed non invasively using diffusion tensor magnetic resonance imaging (DT-MRI). This technique permits the spatial mapping of the apparent diffusion tensor of water $(\mathbf{D})$ in the brain, from which the mean diffusivity $(<\mathrm{D}>)$ and scalar diffusion anisotropy indices, such as the fractional anisotropy (FA), can be determined. ${ }^{45}$ The parameter $\langle\mathrm{D}\rangle$, which is thought to reflect the average microscopic mobility of water molecules in the extravascular extracellular space, ${ }^{6}$ can detect and characterise brain oedema, while FA provides a scalar measure of the deviation from pure isotropic diffusion of water mobility in vivo. ${ }^{5}$ Recent studies have shown that $<\mathrm{D}>$ and FA of peritumoural oedematous brain are respectively increased and reduced compared with normal healthy cerebral tissue. ${ }^{7-9}$ This rise in $\angle \mathrm{D}>$ indicates an increase in the magnitude of water mobility, whereas the decrease in FA suggests a loss of structural organisation within peritumoural brain. These alterations in $\langle\mathrm{D}>$ and FA are thought to arise from a combination of increased water content and tumour infiltration. ${ }^{9}$ If steroids act by producing a normalisation in peritumoural brain water homeostasis, then this action should reduce $\langle\mathrm{D}\rangle$ and increase FA towards the values seen in healthy brain parenchyma. In this paper this hypothesis is investigated by measuring the water diffusion parameters of oedematous and normal brain in a group of patients with intracranial tumours before and $48-72 \mathrm{~h}$ after dexamethasone treatment.

\section{METHODS}

\section{Subjects}

Twenty consecutive patients presenting with a newly diagnosed, supratentorial, solitary, intracranial tumour were enrolled in this prospective study. Each subject had evidence of oedema on the initial CT scan, but without indication of other concurrent neurological disease. At the time of imaging, none of the patients had (i) begun steroid treatment, (ii) had any prior radiotherapy or chemotherapy, or (iii) undergone any prior cranial surgery. They also had no contraindications to MRI. The local ethics committee approved the study and informed consent was obtained from each patient.

\section{MRI protocol}

All MRI data were obtained using a GE Signa LX $1.5 \mathrm{~T}$ (General Electric, Milwaukee, WI, USA) clinical scanner, equipped with a self-shielding gradient set $(22 \mathrm{mT} / \mathrm{m}$ maximum gradient strength and $120 \mathrm{~T} / \mathrm{m} / \mathrm{s}$ slew rate) and a manufacturer-supplied "birdcage" quadrature head coil. The MRI examination consisted of a standard fast spin-echo (FSE) $\mathrm{T}_{2}$-weighted sequence, a DT-MRI protocol described previously, and a contrast-enhanced $\mathrm{T}_{1}$-weighted volume sequence. ${ }^{78}$ The duration of the examination was approximately $40 \mathrm{~min}$. This protocol was repeated $48-72 \mathrm{~h}$ after dexamethasone treatment ( $16 \mathrm{mg} /$ day) had begun.

To ensure that the slice locations used in the second examination corresponded as closely as possible to those in the first, the subject's head position and tilt in the first scan were recorded and the patient repositioned in exactly the same manner for the second scan. At least one of the slices

Abbreviations: DT-MRI, diffusion tensor magnetic resonance imaging; $D W$, diffusion-weighted; EP, echo-planar; $F A$, fractional anisotropy; FOV, field of view; FSE, fast spin-echo; GBM, glioblastoma multiforme; $\mathrm{ROI}$, region of interest; $\mathrm{SD}$, standard deviation 
was taken through a prominent anatomical landmark so as to minimise any deviation in slice location in the second scan. Computational image realignment techniques were then used to warp the images in the second exam to the first, thereby minimising any small remaining positioning errors.

In the DT-MRI experiment diffusion-weighted (DW) images were acquired using a single-shot spin-echo echoplanar (EP) imaging sequence in which two symmetric trapezoidal gradient pulses of duration $\delta=32.2 \mathrm{~ms}$, separation $\Delta=39.1 \mathrm{~ms}$, and rise time $\eta=1.2 \mathrm{~ms}$ were inserted around the $180^{\circ}$ refocusing pulse in the required gradient channel. Sets of axial DW-EP images ( $b=0$ and $1000 \mathrm{~s} / \mathrm{mm}^{2}$ ) were collected with diffusion gradients applied sequentially along six non collinear directions. ${ }^{10}$ Five acquisitions consisting of a baseline $\mathrm{T}_{2}$-weighted EP image $\left(\mathbf{G}^{0}\right)$ and six DW-EP images $\left(\mathbf{G}^{1}\right.$ to $\left.\mathbf{G}^{6}\right)$, a total of 35 images, were collected per slice position. The acquisition parameters for the DW-EP imaging sequence were 15 axial slices of $5 \mathrm{~mm}$ thickness and $1.0 \mathrm{~mm}$ slice gap, a field of view (FOV) of $240 \times 240 \mathrm{~mm}$, an acquisition matrix of $128 \times 128$ (zero filled to $256 \times 256$ ), a TR of $10 \mathrm{~s}$, and a TE of $98.8 \mathrm{~ms}$.

After the DT-MRI protocol, $20 \mathrm{ml}$ of gadopentetate dimeglumine (Magnevist, Berlex Laboratories, Wayne, NJ, USA) was administered intravenously. Acquisition parameters for the contrast enhanced $\mathrm{T}_{1}$-weighted volume sequence were 110 contiguous axial slices of $1.5 \mathrm{~mm}$ thickness, an FOV of $240 \times 240 \mathrm{~mm}$, an acquisition matrix of $256 \times 256$, a TR of $7.3 \mathrm{~ms}$, a TE of $3.2 \mathrm{~ms}$, and a TI of $400 \mathrm{~ms}$.

\section{Image analysis}

Quantitative co-registered maps of brain water diffusion parameters for the pre- and post-treatment examinations were obtained in the following manner. Bulk patient motion and eddy current induced artefacts were removed from the component EP images using FLIRT (www.fmrib.ox.ac.uk/fsl), a three-dimensional computational image alignment program. ${ }^{11}$ Firstly, EP images with the same b-matrix were rigidly aligned to remove patient motion. Secondly, affine transformations were used to align the DW-EP images acquired with diffusion gradient directions $\mathbf{G}^{2}$ to $\mathbf{G}^{6}$ to the first gradient direction $\mathbf{G}^{1}$. Thirdly, all DW-EP images were aligned to the $T_{2}$-weighted $\left(\mathbf{G}^{0}\right)$ EP images acquired in the first examination. Next the set of five EP images collected for each gradient direction was averaged to give seven high signal-to-noise ratio images for each slice. From this MRI data D was calculated in each voxel from the signal intensities in the component EP images. ${ }^{4}$ After diagonalisation of $\mathbf{D}$ to yield the magnitude sorted eigenvalues $\left(\lambda_{i=1,2,3}\right)$, maps of the $\mathrm{T}_{2}$-weighted signal intensity, mean diffusivity

$$
<\mathrm{D}>=\frac{\left(\lambda_{1}+\lambda_{2}+\lambda_{3}\right)}{3},
$$

and the fractional anisotropy ${ }^{5}$

$$
\begin{aligned}
\mathrm{FA}= & \sqrt{\frac{3}{2}} \\
& \sqrt{\frac{\left(\lambda_{1}-<\mathrm{D}>\right)^{2}+\left(\lambda_{2}-<\mathrm{D}>\right)^{2}+\left(\lambda_{3}-<\mathrm{D}>\right)^{2}}{\lambda_{1}^{2}+\lambda_{2}^{2}+\lambda_{3}^{2}}},
\end{aligned}
$$

were generated on a voxel-by-voxel basis and converted into Analyze (Mayo Foundation, Rochester, MN, USA) format. The FA measures the fraction of the total "magnitude" of $\mathbf{D}$ that is anisotropic, and takes a value of 0 for isotropic diffusion $\left(\lambda_{1}=\lambda_{2}=\lambda_{3}\right)$ and $\mathrm{l}$ for completely anisotropic diffusion $\left(\lambda_{1}>0 ; \lambda_{2}=\lambda_{3}=0\right)$.

\section{Region-of-interest analysis}

The effects of dexamethasone on the brain water diffusion parameters of peritumoural oedematous brain were quantified using a previously described region-of-interest (ROI) analysis. ${ }^{7}$ Blinded as to whether the imaging data came from the pre- or post-treatment examination, the observer (SS) identified for each slice the largest region of signal hyperintensity on the $\mathrm{T}_{2}$-weighted EP images which extended beyond the enhancing tumour margin present in the coregistered contrast-enhanced $\mathrm{T}_{1}$-weighted volume images. This oedematous brain region was overlaid on the $<\mathrm{D}>$ and FA parametric maps obtained from both examinations. For each appropriate slice, values of $\langle\mathrm{D}\rangle$ and FA for the entire oedematous brain region and apparently normal contralateral white matter in the centrum semiovale were measured. Overall mean $<\mathrm{D}>$ and FA values were then calculated from these data to give volume measures for the two tissue types in each patient. The volume measurements of $\langle\mathrm{D}\rangle$ and FA were typically obtained from several thousand voxels in five to 12 slices for oedematous brain, and hundreds of voxels in a single slice for apparently normal contralateral white matter in the centrum semiovale.

\section{Statistical analysis}

All data are reported as a mean with one standard deviation (SD) in parentheses. For each patient the percentage change in $\langle\mathrm{D}>$ and FA for oedematous and normal brain following steroid treatment was determined as follows

$$
\varepsilon(X)=100 \frac{X_{\text {post }}-X_{\text {pre }}}{X_{\text {pre }}} \text {, where } X=<D>\text { or FA. }
$$

The percentage change $(\epsilon)$ in $\langle\mathrm{D}\rangle$ and FA for both tissue types was calculated for each patient, and a mean value $(\langle\epsilon\rangle)$ obtained for each of the three disease groups. To assess whether changes in these water diffusion parameters were significant, the mean pre- and post-treatment values of $<\mathrm{D}>$ and FA for each patient were compared using a pairedsamples Student's $t$ test (SPSS 10.0, SPSS, Chicago, IL, USA), with $\mathrm{p}<0.05$ being considered statistically significant.

\section{RESULTS}

Of the 20 patients initial enrolled in the study, seven men and eight women (mean (SD) age 58.6 (9.7) years) tolerated both examinations and had an enhancing lesion on the $\mathrm{T}_{1}$ weighted volume scan. Of these 15 patients, seven had glioblastoma multiforme (GBM), four had metastatic carcinoma, and four had meningioma. In all patients the tumour type was confirmed histologically, with all surgical procedures performed after the second MRI examination. There was a significant improvement in limb function in one of the seven patients with GBM following steroid therapy, while in the remaining subjects improvement was more limited and subjective, with the typical benefit being relief from headache.

Pre- and post-treatment values of $\langle\mathrm{D}\rangle$ and $\mathrm{FA}$ for oedematous and normal brain in these patients are shown in table 1 , along with their demographic information. Fig 1 shows maps of $T_{2}$-weighted signal intensity, $\langle D\rangle$, and $F A$ before and after steroid treatment for a representative slice acquired from a 55 year old female patient with GBM (patient 1). These maps show the marked difference in the water diffusion parameters between oedematous and normal brain, with the former characterised by high values of $<\mathrm{D}>$ and low values of FA. This figure also shows that although the water diffusion abnormalities of oedematous brain are not completely resolved by steroid treatment after $72 \mathrm{~h}$, there is a widespread reduction in the $\mathrm{T}_{2}$-weighted signal intensity 

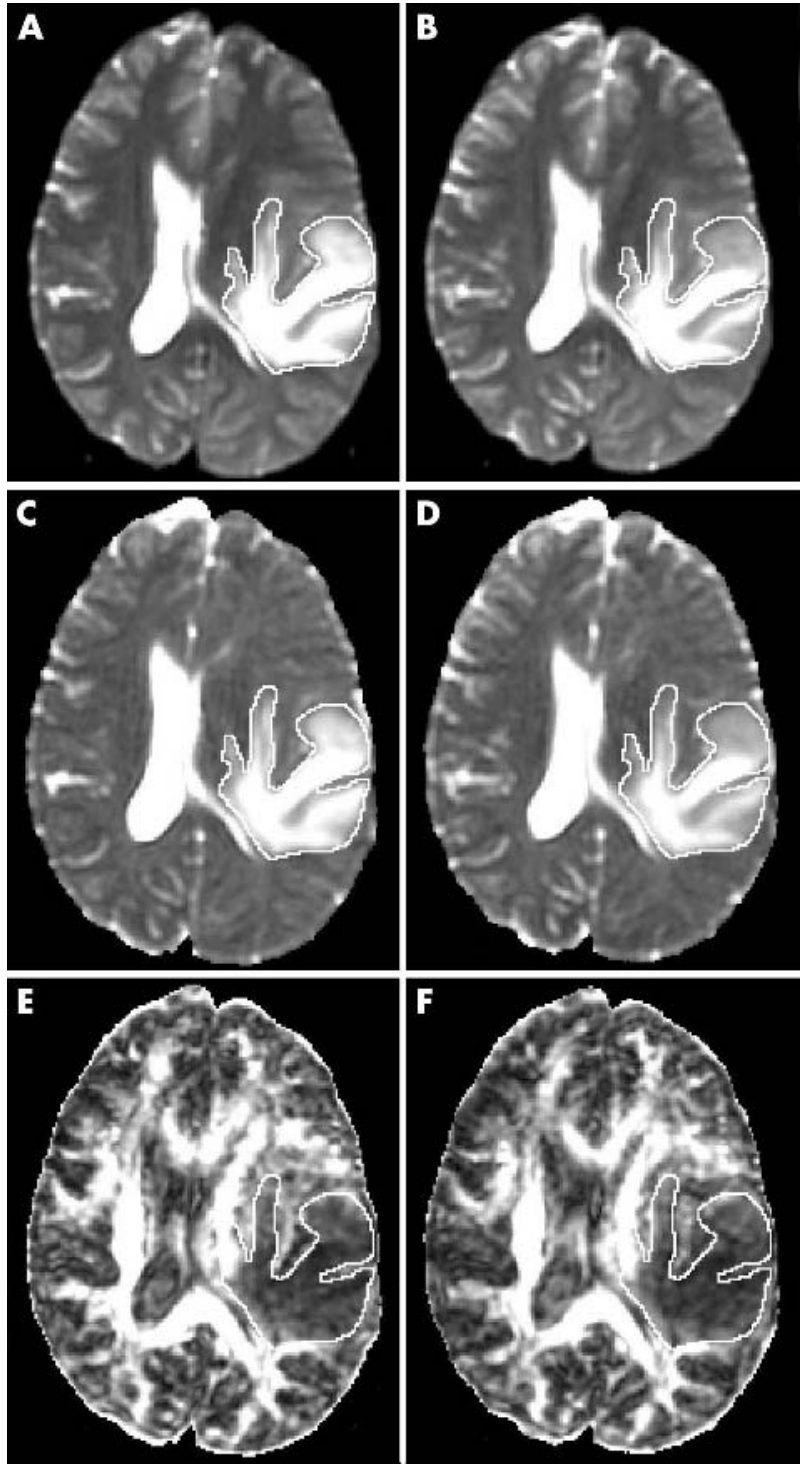

Figure 1 Maps of $\mathrm{T}_{2}$-weighted signal intensity $(\mathrm{A}, \mathrm{B}), \angle \mathrm{D}>(\mathrm{C}, \mathrm{D})$, and FA $(E, F)$ obtained at the level of the body of the lateral ventricles from a 55 year old female patient with GBM (patient 1) before (first column) and $72 \mathrm{~h}$ after steroid treatment. Note the widespread reduction in the $\mathrm{T}_{2}$-weighted signal intensity and $\angle \mathrm{D}>$ of peritumoural oedematous brain after steroid treatment. No enhancing lesion is visible at this level in the brain in the contrast-enhanced $\mathrm{T}_{1}$-weighted volume scan (not shown).

and $<\mathrm{D}>$ of oedematous brain. There is, however, no apparent change in FA of oedematous brain after steroid treatment.

Table 1 shows that $\langle\mathrm{D}\rangle$ of oedematous brain is significantly higher than that of normal brain both before and after steroid treatment in all three patient groups $(p<0.001)$. Conversely, FA of oedematous brain is significantly reduced compared with normal brain before and after treatment $(p<0.001)$. In all three patient groups, there was a significant decrease in $<\mathrm{D}>$ of oedematous brain after steroid treatment $(\mathrm{p}<0.01)$. The mean percentage change $(\langle\epsilon>)$ in $\langle D\rangle$ was -7.63 (1.17) \% for high-grade glioma, -5.73 (1.04) \% for metastatic carcinoma and $-5.54(0.70) \%$ for meningioma. The FA of oedematous brain was not, however, significantly altered by steroid treatment in any of the three disease groups. In addition, there was no significant change in either $<\mathrm{D}>$ or FA of apparently normal contralateral white matter after treatment.

\section{DISCUSSION}

There have been several recent studies using DT-MRI to characterise the water diffusion properties of intracranial tumours. Sinha et al investigated whether water diffusion parameters could differentiate tumour margin from oedematous brain in nine patients with GBM. They found that while $<\mathrm{D}>$ of tumour margin was significantly different from that of oedematous brain, FA was not. Lu et al ${ }^{9}$ measured $<\mathrm{D}>$ and FA values in peritumoural brain in 12 patients with highgrade glioma and 12 with metastatic lesions and found that only $<\mathrm{D}>$ was significantly different in these two pathologies. Price et $a^{12}$ found evidence for tumour infiltration in high-grade gliomas, when comparing DT-MRI and $\mathrm{T}_{2}-$ weighted imaging data from 20 patients with gliomas and metastases. By analysing MRI and biopsy data in 31 patients with astrocytic tumours, Beppu et al ${ }^{13}$ found evidence that the FA values of glioblastoma and anaplastic, diffuse, and pilocytic astrocytoma are largely affected by cellularity and/ or vascularity. Changes in the water diffusion parameters of oedematous brain following dexamethasone treatment have previously only been described by Bastin et al. ${ }^{14}$ In their small pilot study of six patients with a wide range of tumours, they found evidence for a reduction in $\langle\mathrm{D}\rangle$ of oedematous brain following dexamethasone treatment in one patient with GBM.

The results of the current study show that $\langle\mathrm{D}\rangle$ of oedematous brain associated with high-grade glioma, metastatic carcinoma, and meningioma is significantly reduced 48-72 $\mathrm{h}$ after dexamethasone treatment, and that this reduction is of a similar magnitude in all three tumour types. Previous studies have shown that oedematous brain associated with intra- and extra-axial tumours has a much higher proton longitudinal relaxation time $\left(\mathrm{T}_{1}\right)$ than normal brain, and that dexamethasone reduces these elevated $\mathrm{T}_{1}$ values. ${ }^{8}{ }^{15-17}$ Since $T_{1}$ reflects brain tissue water content, and DT-MRI predominantly measures the mobility of water molecules in the extracellular space, ${ }^{6}$ these data suggest that dexamethasone acts by reducing the extracellular water fraction. This reduction then produces the marked decline in $\langle\mathrm{D}\rangle$ of oedematous brain observed above. Interestingly, however, this effect is not sufficient, at least after $48-72 \mathrm{~h}$, to increase axonal organisation, as measured by FA, towards more normal values. Furthermore, since significant differences in $\langle\mathrm{D}>$ and FA were not seen in normal brain, it is concluded that dexamethasone has only a localised effect on brain water content.

The current study has both strengths and weaknesses. Firstly, none of the patients had undergone previous treatment or had recurrent and/or residual tumours. Since the effect of surgery or adjunct therapy on oedematous brain $<\mathrm{D}>$ and FA values is not known, excluding such patients makes quantifying the changes in water diffusion parameters after steroid treatment more straightforward. Secondly, by measuring the water diffusion parameters over the entire oedematous brain volume one avoids any subjective bias that may occur when small ROIs are placed in regions of abnormal signal intensity. ${ }^{7}$ The main limitation of the paper is the small number of patients imaged. However, even with this number of subjects the effects of dexamethasone on the water diffusion parameters of peritumoural oedematous brain are clear.

\section{CONCLUSION}

In this study the effects of dexamethasone on the water diffusion parameters of oedema associated with intracranial tumours and apparently normal contralateral white matter have been quantified using DT-MRI. In all three patient groups, $<\mathrm{D}>$ of peritumoural oedematous brain was significantly reduced $48-72 \mathrm{~h}$ following steroid treatment, 
Table 1 Values of the mean diffusivity $(\langle D>$ ) and fractional anisotropy (FA) for peritumoural oedematous brain and apparently normal contralateral white matter (centrum semiovale) pre- and post-steroid treatment for the 15 patients imaged in this study

\begin{tabular}{|c|c|c|c|c|c|c|c|c|}
\hline \multirow[b]{3}{*}{ Age/sex } & \multicolumn{4}{|c|}{ Mean diffusivity, $<\mathrm{D}>\left(\times 10^{-6} \mathrm{~mm}^{2} / \mathrm{s}\right)$} & \multicolumn{4}{|c|}{ Fractional anisotropy } \\
\hline & \multicolumn{2}{|c|}{ Oedematous brain } & \multicolumn{2}{|c|}{ Contralateral white matter } & \multicolumn{2}{|c|}{ Oedematous brain } & \multicolumn{2}{|c|}{ Contralateral white matter } \\
\hline & Pre-steroids & Post-steroids & Pre-steroids & Post-steroids & Pre-steroids & Post-steroids & Pre-steroids & Post-steroids \\
\hline \multicolumn{9}{|l|}{ Glioblastoma } \\
\hline $1 / 55 / F$ & $1674(253)$ & $1561(318)$ & $750(18)$ & $740(24)$ & $0.15(0.06)$ & $0.16(0.07)$ & $0.47(0.10)$ & $0.47(0.10)$ \\
\hline $2 / 50 / M$ & $1575(215)$ & $1430(256)$ & $758(24)$ & $766(22)$ & $0.15(0.05)$ & $0.17(0.07)$ & $0.49(0.05)$ & $0.48(0.04)$ \\
\hline $3 / 61 / F$ & $1139(152)$ & 1046 (144) & $719(25)$ & $716(22)$ & $0.21(0.08)$ & $0.21(0.09)$ & $0.43(0.05)$ & $0.42(0.04)$ \\
\hline $4 / 65 / M$ & $1494(211)$ & 1377 (228) & $717(46)$ & $711(54)$ & $0.14(0.05)$ & $0.15(0.06)$ & $0.45(0.13)$ & $0.46(0.14)$ \\
\hline $5 / 71 / M$ & $1477(280)$ & $1347(289)$ & $775(63)$ & $733(24)$ & $0.18(0.09)$ & $0.19(0.10)$ & $0.52(0.07)$ & $0.50(0.10)$ \\
\hline $6 / 42 / M$ & $1140(336)$ & 1068 (288) & $690(25)$ & 704 (22) & $0.22(0.10)$ & $0.20(0.09)$ & $0.42(0.08)$ & $0.41(0.07)$ \\
\hline $7 / 75 / M$ & $1288(189)$ & $1206(189)$ & $775(75)$ & $767(61)$ & $0.20(0.07)$ & $0.19(0.07)$ & $0.42(0.13)$ & $0.41(0.10)$ \\
\hline Mean & $1398(212)$ & 1291 (191) & $741(33)$ & $734(26)$ & $0.18(0.03)$ & $0.18(0.02)$ & $0.46(0.04)$ & $0.45(0.04)$ \\
\hline$<\epsilon>, \% ; p$ & \multicolumn{2}{|c|}{$-7.63(1.17) ;<0.01$} & \multicolumn{2}{|c|}{$-0.85(2.35) ; 0.36$} & \multicolumn{2}{|c|}{$3.12(8.48) ; 0.55$} & \multicolumn{2}{|c|}{$-1.50(2.11) ; 0.11$} \\
\hline \multicolumn{9}{|l|}{ Metastases } \\
\hline $8 / 54 / F^{*}$ & $1356(231)$ & $1264(216)$ & $676(24)$ & $696(31)$ & $0.18(0.07)$ & $0.19(0.08)$ & $0.41(0.09)$ & $0.44(0.09)$ \\
\hline 9/59/F† & $1554(244)$ & $1460(224)$ & $721(52)$ & $733(55)$ & $0.14(0.06)$ & $0.13(0.06)$ & $0.42(0.12)$ & $0.39(0.12)$ \\
\hline $10 / 61 / M \ddagger$ & $1560(268)$ & $1470(274)$ & $734(42)$ & $728(33)$ & $0.13(0.06)$ & $0.13(0.07)$ & $0.48(0.10)$ & $0.49(0.08)$ \\
\hline $11 / 53 / F^{*}$ & $1556(236)$ & $1489(216)$ & $764(22)$ & $724(24)$ & $0.15(0.07)$ & $0.14(0.07)$ & $0.49(0.07)$ & $0.48(0.11)$ \\
\hline Mean & $1507(100)$ & 1421 (105) & $724(37)$ & $720(17)$ & $0.15(0.02)$ & $0.15(0.03)$ & $0.45(0.04)$ & $0.45(0.05)$ \\
\hline$<\epsilon>, \% ; p$ & \multicolumn{2}{|c|}{$-5.73(1.04) ;<0.01$} & \multicolumn{2}{|c|}{$-0.36(3.60) ; 0.81$} & \multicolumn{2}{|c|}{$-2.36(4.13) ; 0.37$} & \multirow{2}{*}{\multicolumn{2}{|c|}{$-0.20(5.36) ; 0.94$}} \\
\hline \multicolumn{7}{|l|}{ Meningioma } & & \\
\hline $12 / 60 / F$ & $1442(224)$ & 1371 (219) & $742(14)$ & $732(12)$ & $0.16(0.06)$ & $0.16(0.07)$ & $0.46(0.06)$ & $0.45(0.07)$ \\
\hline $13 / 52 / \mathrm{F}$ & $1215(183)$ & 1155 (166) & $720(14)$ & $723(20)$ & $0.24(0.08)$ & $0.23(0.07)$ & $0.44(0.08)$ & $0.44(0.08)$ \\
\hline 14/74/M & $1077(140)$ & 1011 (141) & $741(45)$ & $778(43)$ & $0.31(0.10)$ & $0.28(0.10)$ & $0.45(0.09)$ & $0.44(0.08)$ \\
\hline $15 / 47 / F$ & 1539 (249) & 1444 (241) & 723 (37) & 722 (35) & $0.13(0.06)$ & $0.14(0.06)$ & $0.38(0.13)$ & $0.38(0.12)$ \\
\hline Mean & $1318(210)$ & 1245 (199) & 732 (12) & $739(27)$ & $0.21(0.08)$ & $0.20(0.07)$ & $0.43(0.04)$ & $0.43(0.04)$ \\
\hline$<\epsilon>, \% ; p$ & \multicolumn{2}{|c|}{$-5.54(0.70) ;<0.01$} & \multicolumn{2}{|c|}{$0.98(2.77) ; 0.53$} & \multicolumn{2}{|c|}{$-2.90(5.09) ; 0.34$} & \multicolumn{2}{|c|}{$-0.49(2.12) ; 0.63$} \\
\hline
\end{tabular}

while FA was approximately unchanged. The water diffusion parameters of normal tissue were also unchanged. These data indicate that dexamethasone produces a localised reduction in the magnitude of extracellular water molecule mobility, and hence water content, in peritumoural oedematous brain. Furthermore, the magnitude of these changes is similar for both intra- and extra-axial tumours. These results also indicate that DT-MRI may provide a sensitive non invasive tool for evaluating the treatment response of peritumoural oedema not only to dexamethasone, but potentially also to other chemotherapeutic agents.

\section{ACKNOWLEDGEMENTS}

This work was undertaken at the SHEFC Brain Imaging Research Centre for Scotland, Edinburgh, UK (http://www.dcn.ed.ac.uk/bic).

\section{Authors' affiliations \\ S Sinha, J M Wardlaw, P A Armitage, I R Whittle, Clinical EH4 2XU, UK \\ M E Bastin, Medical and Radiological Sciences (Medical Physics), University of Edinburgh, Western General Hospital, Crewe Road, Edinburgh, EH4 2XU, UK \\ This work was funded by the Cunningham Trust. \\ Competing interests: none declared \\ SS and MEB authors contributed equally to this work. \\ IW was not involved in the review process.}

Neurosciences, Western General Hospital, Crewe Road, Edinburgh,

Correspondence to: Dr M E Bastin, Medical and Radiological Sciences (Medical Physics), University of Edinburgh, Western General Hospital, Crewe Road, Edinburgh, EH4 2XU, UK; meb@skull.den.ed.ac.uk

Received 18 September 2003

In revised form 20 January 2004

Accepted 20 January 2004

\section{REFERENCES}

1 Galicich JH, French LA, Melby JC. Use of dexamethasone in the treatment of cerebral edema associated with brain tumours. Lancet 1961;81:46-53.

2 Maxwell RE, Long DM, French LA. The clinical effects of a synthetic glucocorticoid used for brain edema in the practice of neurosurgery. In: Reulen $\mathrm{HJ}$, Schurmann K, eds. Steroids and brain edema. Berlin: Springer, 1972:219-32.

3 Swaroops GR, Kelly PA, Holmes MC, et al. The effects of dexamethasone therapy on permeability, blood flow and iNOS expression in experimental glioma. J Clin Neurosci 2001;8:35-9.

4 Basser PJ, Mattiello J, LeBihan D. Estimation of the effective self-diffusion tensor from the NMR spin echo. J Magn Reson B 1994;103:247-54.

5 Basser PJ. Inferring microstructural features and the physiological state of tissues from diffusion-weighted images. NMR Biomed 1995;8:333-44.

6 Inglis BA, Bossart EL, Buckley DL, et al. Visualization of neural tissue water compartments using biexponential diffusion tensor MRI. Magn Reson Med 2001;45:580-7.

7 Sinha S, Bastin ME, Whittle IR, et al. Diffusion tensor MR imaging of highgrade cerebral gliomas. AJNR Am J Neuroradiol 2002;23:520-7.

8 Bastin ME, Sinha S, Whittle IR, et al. Measurements of water diffusion and T1 values in peritumoural oedematous brain. Neuroreport 2002;13:1335-40.

9 Lu S, Ahn D, Johnson G, et al. Peritumoral diffusion tensor imaging of highgrade gliomas and metastatic brain tumors. AJNR Am J Neuroradiol 2003;24:937-41.

10 Basser PJ, Pierpaoli C. A simplified method to measure the diffusion tensor from seven MR images. Magn Reson Med 1998;39:928-34.

11 Jenkinson M, Smith S. A global optimisation method for robust affine registration of brain images. Med Image Anal 2001;5:143-56.

12 Price SJ, Burnet NG, Donovan T, et al. Diffusion tensor imaging of brain tumours at 3T: a potential tool for assessing white matter tract invasion? Clin Radiol 2003;58:455-62.

13 Beppu T, Inoue T, Shibata Y, et al. Measurement of fractional anisotropy using diffusion tensor MRI in supratentorial astrocytic tumors. J Neurooncol 2003;63:109-16.

14 Bastin ME, Delgado M, Whittle IR, et al. The use of diffusion tensor imaging in quantifying the effect of dexamethasone on brain tumours. Neuroreport 1999; 10:1385-91

15 Andersen C, Haselgrove JC, Doenstrup S, et al. Resorption of peritumoural oedema in cerebral gliomas during dexamethasone treatment evaluated by NMR relaxation time imaging. Acta Neurochir 1993;122:218-24.

16 Andersen C, Astrup J, Gyldensted C. Quantitative MR analysis of glucocorticoid effects on peritumoral edema associated with intracranial meningiomas and metastases. J Comput Assist Tomogr 1994;18:509-18.

17 Andersen C, Jensen FT. Differences in blood-tumour-barrier leakage of human intracranial tumours: quantitative monitoring of vasogenic oedema and its response to glucocorticoid treatment. Acta Neurochir 1998; 140:919-24. 